\title{
$B$ fields in OB stars (BOB): Concluding the FORS 2 observing campaign ${ }^{\star}$
}

\author{
M. Schöller ${ }^{1}$, S. Hubrig ${ }^{2}$, L. Fossati ${ }^{3,4}$, T. A. Carroll ${ }^{2}$, M. Briquet ${ }^{5,2}$, L. M. Oskinova ${ }^{6}$, S. Järvinen ${ }^{2}$, I. Ilyin ${ }^{2}$, \\ N. Castro ${ }^{7,4}$, T. Morel $^{5}$, N. Langer ${ }^{4}$, N. Przybilla ${ }^{8}$, M.-F. Nieva ${ }^{8}$, A. F. Kholtygin ${ }^{9}$, H. Sana ${ }^{10}$, A. Herrero ${ }^{11,12}$, \\ R. H. Barbá ${ }^{13}$, A. de Koter ${ }^{14}$, and the BOB Collaboration \\ ${ }^{1}$ European Southern Observatory, Karl-Schwarzschild-Str. 2, 85748 Garching, Germany \\ e-mail: mschoell@eso.org \\ 2 Leibniz-Institut für Astrophysik Potsdam (AIP), An der Sternwarte 16, 14482 Potsdam, Germany \\ 3 Österreichische Akademie der Wissenschaften, Institut für Weltraumforschung, Schmiedlstraße 6, 8042 Graz, Austria \\ 4 Argelander-Institut für Astronomie der Universität Bonn, Auf dem Hügel 71, 53121 Bonn, Germany \\ 5 Space Sciences, Technologies and Astrophysics Research (STAR) Institute, Université de Liège, Quartier Agora, \\ Allée du 6 Août 19c, Bât. B5C, 4000 Liège, Belgium \\ ${ }^{6}$ Universität Potsdam, Institut für Physik und Astronomie, Karl-Liebknecht-Str. 24/25, 14476 Potsdam, Germany \\ 7 Department of Astronomy, University of Michigan, 1085 S. University Avenue, Ann Arbor, MI 48109-1107, USA \\ 8 Institut für Astro- und Teilchenphysik, Universität Innsbruck, Technikerstr. 25/8, 6020 Innsbruck, Austria \\ 9 St. Petersburg State University, Universitetski pr. 28, 198504 St. Petersburg, Russia \\ 10 Institute of Astrophysics, KU Leuven, Celestijnlaan 200D, 3001 Leuven, Belgium \\ 11 Instituto de Astrofísica de Canarias, C/vía Láctea s/n, 38205 La Laguna, Spain \\ 12 Universidad de La Laguna, Departamento de Astrofísica, Avda. Francisco Sanchez 2, 38206 La Laguna, Spain \\ 13 Departamento de Física y Astronomía, Universidad de La Serena, Av. Juan Cisternas 1200 Norte, La Serena, Chile \\ 14 Anton Pannekoek Institute for Astronomy, University of Amsterdam, Science Park 904, PO Box 94249, 1090 GE Amsterdam, \\ The Netherlands
}

Received 12 May 2016 / Accepted 11 November 2016

\section{ABSTRACT}

\begin{abstract}
Aims. The $B$ fields in OB stars (BOB) Collaboration is based on an ESO Large Programme to study the occurrence rate, properties, and ultimately the origin of magnetic fields in massive stars.

Methods. In the framework of this program, we carried out low-resolution spectropolarimetric observations of a large sample of massive stars using FORS 2 installed at the ESO VLT $8 \mathrm{~m}$ telescope.

Results. We determined the magnetic field values with two completely independent reduction and analysis pipelines. Our in-depth study of the magnetic field measurements shows that differences between our two pipelines are usually well within $3 \sigma$ errors. From the 32 observations of $28 \mathrm{OB}$ stars, we were able to monitor the magnetic fields in CPD $-57^{\circ} 3509$ and HD $164492 \mathrm{C}$, confirm the magnetic field in HD 54879, and detect a magnetic field in CPD $-62^{\circ} 2124$. We obtain a magnetic field detection rate of $6 \pm 3 \%$ for the full sample of $69 \mathrm{OB}$ stars observed with FORS 2 within the BOB program. For the preselected objects with a $v$ sin $i$ below $60 \mathrm{~km} \mathrm{~s}^{-1}$, we obtain a magnetic field detection rate of $5 \pm 5 \%$. We also discuss X-ray properties and multiplicity of the objects in our FORS 2 sample with respect to the magnetic field detections.
\end{abstract}

Key words. polarization - stars: early-type - stars: magnetic field - stars: massive

\section{Introduction}

Magnetic fields may have a key influence on the evolution of massive stars. Magnetized winds can spin down the star by applying a torque at the surface (ud-Doula et al. 2008; Meynet et al. 2011) and can mediate angular momentum transport in the stellar interior (Heger et al. 2005; Maeder \& Meynet 2005). Understanding these processes will provide strong constraints on the role of rotationally induced mixing in massive stars (Brott et al. 2011), and on the frequency of long gamma-ray bursts (Yoon et al. 2006) and massive black hole mergers (Mandel \& de Mink 2016; Marchant et al. 2016). Dedicated surveys targeting the detection and characterization of magnetic fields in massive stars

\footnotetext{
^ Based on observations made with ESO Telescopes at the La Silla Paranal Observatory under program ID 191.D-0255(E,G).
}

have only started in recent years (Wade et al. 2016; Morel et al. $2014,2015)$, leading to an increase in the number of massive stars with characterized magnetic fields.

The $B$ fields in OB stars (BOB) Collaboration, established in 2013, aims at investigating the frequency and strength distribution of magnetic fields in OB stars using spectropolarimetric observations, concentrating mainly on slow rotators. Data are obtained in the framework of the ESO Large Programme 191.D-0255, scheduled on the $8 \mathrm{~m}$ Very Large Telescope (VLT) on Paranal and the ESO $3.6 \mathrm{~m}$ telescope on La Silla. The instruments used for the spectropolarimetric observations are the FOcal Reducer low dispersion Spectrograph (FORS 2; Appenzeller et al. 1998) and the High Accuracy Radial velocity Planet Searcher polarimeter (HARPSpol; Snik et al. 2008). Details about the aims and implications of the first discoveries by the 
Table 1. Objects studied with FORS 2 during the runs in 2014 June and 2015 March.

\begin{tabular}{lcr}
\hline \hline \multicolumn{1}{c}{ Object } & Spectral type & \multicolumn{1}{c}{$m_{\mathrm{V}}$} \\
\hline BD -12 4982 & B0 II & 9.22 \\
CD -22 12513 & B0 V & 10.01 \\
CPD -57 3509* & B2 IV & 10.68 \\
CPD -62 2124 & B2 IV & 10.99 \\
HD 54879* & O9.7 V & 7.64 \\
HD 56779 & B2 IV-V & 5.02 \\
HD 72754* & B2Ia.pshe & 6.85 \\
HD 75759 & O9 V + B0 V & 5.99 \\
HD 95568* & O9/B0 V & 9.60 \\
HD 97991* & B2 II & 7.40 \\
HD 110432 & B2 pe & 5.31 \\
HD 118198* & O9.7 III & 8.47 \\
HD 120324 & B2 Vnpe & 3.35 \\
HD 120991 & B2 IIne & 6.08 \\
HD 156134 & B0 Ib & 8.06 \\
HD 156233 & B0 & 9.10 \\
HD 156234 & B0 III & 7.74 \\
HD 156292 & O9.7 III & 7.51 \\
HD 164492B & B2 Vnn & 10.52 \\
HD 164492C* & B1 V & 8.66 \\
HD 164536 & O7.5 V(n)z & 7.11 \\
HD 164704 & B2 II & 8.16 \\
HD 164816 & O9.5 V + B0 V & 7.09 \\
HD 164844 & B1/B2 III & 8.29 \\
HD 165052 & O7 Vz + O7.5 Vz & 6.87 \\
HD 166033 & B1 V & 9.60 \\
HD 315032 & B2 Vne & 9.19 \\
HD 345439 & B1/B2 V & 11.26 \\
\hline
\end{tabular}

Notes. Objects indicated with an asterisk were also observed in runs A and C (Fossati et al. 2015).

BOB survey are presented by Morel et al. $(2014,2015)$ and Fossati et al. (2015).

In this work, we present the most recent FORS 2 observations of 28 stars, concluding the low-resolution spectropolarimetric campaign within the BOB survey. We describe the observations and data reduction in Sect. 2, present the magnetic field measurements in Sect. 3, give an extensive overview about the statistics and quality of the measurements in Sect. 4, discuss some object properties in Sect. 5, and finally conclude with a discussion in Sect. 6.

\section{Observations and data reduction}

The BOB Collaboration mainly targets OB dwarfs and giants with low projected rotation velocities. A full description of the target selection criteria was presented by Fossati et al. (2015), who published FORS 2 magnetic field measurements of $50 \mathrm{O}-$ and early B-type stars obtained in runs A and C. Since the presence of weak magnetic fields in Be-type stars is not yet established, we additionally carried out observations of four $\mathrm{Be}$ stars, three of which were selected on the basis of their hard X-ray spectra (see Sect. 5.3).

We conducted 32 new spectropolarimetric observations of $28 \mathrm{OB}$ stars in visitor mode with FORS 2 during 2.5 nights each on 2014 June 1-3 and 2015 March 16-18, as part of runs E and $\mathrm{G}$ of our observing campaign. No data were obtained on 2015 March 16 and 18, because of high humidity and clouds, respectively. All other nights had severe issues with clouds or strong winds. The observed objects are listed in Table 1, together with their spectral type and their $V$ magnitude. The main sources of the spectral classification are the Galactic O-Star Spectroscopic Survey (Sota et al. 2014), the Michigan Catalogue of HD stars (see Houk 1994), and previous BOB publications. The primary source for the photometry is the catalog of Mermilliod (2006). For seven of these objects, observations were already carried out in earlier runs with FORS 2 and were reported by Fossati et al. (2015). We reobserved these sources either to check earlier marginal magnetic field detections or to obtain magnetic field measurements at different phases of the, usually unknown, rotation cycle of the object. In total, 71 different objects were observed with FORS 2 within the BOB program, in 134 observations.

FORS 2 is a multimode instrument equipped with polarization-analyzing optics comprising super-achromatic halfwave and quarter-wave phase retarder plates, and a Wollaston prism with a beam divergence of $22^{\prime \prime}$ in standard-resolution mode. We used the GRISM 600B and the narrowest available slit width of 0 !'4 to obtain a spectral resolving power of $R \sim 2000$. The observed spectral range from 3250 to $6215 \AA$ includes all Balmer lines apart from $\mathrm{H} \alpha$, and numerous He I lines. The position angle of the retarder waveplate was alternated in the sequence $-45^{\circ}+45^{\circ}+45^{\circ}-45^{\circ}$. Between two to six such sequences of exposures were combined to form a single polarimetric observation. For the observations, we used a non-standard readout mode with low gain $(200 \mathrm{kHz}, 1 \times 1$,low $)$, which provides a broader dynamic range, hence allowing us to reach a higher signal-to-noise ratio $(\mathrm{S} / \mathrm{N})$ in the individual exposures, which is especially advantageous for bright targets.

The determination of the mean longitudinal magnetic field using low-resolution FORS $1 / 2$ spectropolarimetry has been described in detail by two different groups (Bagnulo et al. 2002, 2009, 2012; Hubrig et al. 2004a, b, 2016; Schöller et al., in prep.). The $V / I$ spectrum is calculated using

$\frac{V}{I}=\frac{1}{2}\left\{\left(\frac{f^{\mathrm{o}}-f^{\mathrm{e}}}{f^{\mathrm{o}}+f^{\mathrm{e}}}\right)_{-45^{\circ}}-\left(\frac{f^{\mathrm{o}}-f^{\mathrm{e}}}{f^{\mathrm{o}}+f^{\mathrm{e}}}\right)_{+45^{\circ}}\right\}$,

where $+45^{\circ}$ and $-45^{\circ}$ indicate the position angle of the quarterwave plate and $f^{\circ}$ and $f^{\mathrm{e}}$ are the fluxes of the ordinary and extraordinary beams, respectively.

For low-resolution FORS 2 spectra, the mean longitudinal magnetic field $\left\langle B_{z}\right\rangle$ is usually diagnosed from the slope of a linear regression of $V / I$ versus the quantity $-g_{\mathrm{eff}} \Delta \lambda_{z} \lambda^{2} \frac{1}{I} \frac{\mathrm{d} I}{\mathrm{~d} \lambda}\left\langle B_{z}\right\rangle+$ $V_{0} / I_{0}$, where $V$ is the Stokes parameter that measures the circular polarization, $I$ is the intensity observed in unpolarized light, $g_{\text {eff }}$ is the effective Landé factor, $\lambda$ is the wavelength, $\mathrm{d} I / \mathrm{d} \lambda$ is the derivative of Stokes $I$, and $V_{0} / I_{0}$ denotes the instrumental polarization. The diagnostic $\left\langle N_{z}\right\rangle$ parameter was calculated following the formalism of Bagnulo et al. (2009).

To identify systematic differences that might exist when the FORS 2 data are treated by different groups, the mean longitudinal magnetic field, $\left\langle B_{z}\right\rangle$, was derived for all stars by two groups separately, using independent reduction packages. Details of and differences between the two reduction and analysis packages are described in detail by Fossati et al. (2015).

\section{Results}

We present the results of our magnetic field measurements in Table 2. The first column gives the object name, followed by the modified Julian date, the number of exposures and the total exposure time in Col. 2, and the peak value of the $\mathrm{S} / \mathrm{N}$ for the extracted full Stokes $I$ spectrum, separately for the two groups, 
M. Schöller et al.: $B$ fields in OB stars (BOB): Concluding the FORS 2 observing campaign

Table 2. Results from our spectropolarimetric observations with FORS 2 in 2014 June and 2015 March.

\begin{tabular}{|c|c|c|c|c|c|c|c|}
\hline \multirow[t]{2}{*}{ Object } & \multirow{2}{*}{$\begin{array}{c}\text { MJD } \\
n_{\exp } / t_{\exp }[\mathrm{s}]\end{array}$} & \multirow[t]{2}{*}{$S / N$} & \multirow[t]{2}{*}{ Group } & \multicolumn{2}{|c|}{$\mathrm{H}$ lines } & \multicolumn{2}{|c|}{ Whole spectrum } \\
\hline & & & & $\left\langle B_{z}\right\rangle[\mathrm{G}]$ & $\left\langle N_{z}\right\rangle[\mathrm{G}]$ & $\left\langle B_{z}\right\rangle[\mathrm{G}]$ & $\left\langle N_{z}\right\rangle[\mathrm{G}]$ \\
\hline \multirow[t]{2}{*}{ HD 72754} & 56809.94942 & 3416 & Potsdam & $-50 \pm 36 / 1.4$ & $-29 \pm 33 / 0.9$ & $-29 \pm 27 / 1.1$ & $-16 \pm 24 / 0.7$ \\
\hline & $24 / 320$ & 3308 & Bonn & $-53 \pm 26 / 2.0$ & $-36 \pm 23 / 1.6$ & $-17 \pm 16 / 1.1$ & $-41 \pm 15 / 2.7$ \\
\hline \multirow[t]{2}{*}{ HD 72754} & 56810.94153 & 2906 & Potsdam & $-65 \pm 47 / 1.4$ & $17 \pm 28 / 0.6$ & $-55 \pm 30 / 1.8$ & $31 \pm 20 / 1.6$ \\
\hline & $12 / 203$ & 2779 & Bonn & 28 / 1.4 & $-24 \pm 25 / 1.0$ & $-21 \pm 18 / 1.2$ & $14 \pm 17 / 0.8$ \\
\hline \multirow[t]{2}{*}{$\mathrm{CPD}-57^{\circ} 3509$} & 56809.97981 & 2025 & Potsdam & $=71 / 0.3$ & $-28 \pm 86 / 0.3$ & $88 \pm 54 / 1.6$ & $-45 \pm 59 / 0.8$ \\
\hline & $8 / 4400$ & 1943 & Bonn & $-71 \pm 71 / 1.0$ & $-58 \pm 77 / 0.8$ & $40 \pm 46 / 0.9$ & $-51 \pm 47 / 1.1$ \\
\hline \multirow[t]{2}{*}{ CPD $-57^{\circ} 3509$} & 56810.96178 & 2348 & otsdam & $979 \pm 68 / 14.4$ & $-108 \pm 77 / 1.4$ & $920 \pm 48 / 19.2$ & $2 \pm 50 / 0.0$ \\
\hline & $8 / 4400$ & 2289 & Bonn & $1049 \pm 69 / 15.2$ & $-90 \pm 61 / 1.5$ & $943 \pm 41 / 23.0$ & $1 \pm 39 / 0.0$ \\
\hline \multirow[t]{2}{*}{$\mathrm{CPD}-57^{\circ} 3509$} & 57099.06734 & 1826 & Potsdam & $582 \pm 99 / 5.9$ & $-75 \pm 101 / 0.7$ & $671 \pm 62 / 10.8$ & $-33 \pm 61 / 0.5$ \\
\hline & $8 / 4500$ & 1791 & Bonn & $607 \pm 98 / 6.2$ & $-4 \pm 89 / 0.0$ & $734 \pm 56 / 13.1$ & $8 \pm 55 / 0.1$ \\
\hline \multirow[t]{2}{*}{ HD 118198} & 56810.04647 & 2628 & Potsdam & $-36 \pm 60 / 0.6$ & $46 \pm 68 / 0.7$ & $-84 \pm 34 / 2.5$ & $-14 \pm 38 / 0.4$ \\
\hline & $8 / 800$ & 2583 & 3onn & $-65 \pm 58 / 1.1$ & $13 \pm 57 / 0.2$ & $-66 \pm 37 / 1.8$ & $-43 \pm 37 / 1.2$ \\
\hline HD $164492 C$ & 56810.06846 & 2829 & Potsdam & $651 \pm 85 / 7.7$ & $=73 / 0.7$ & $540 \pm 72 / 7.5$ & $-59 \pm 49 / 1.2$ \\
\hline & $8 / 1520$ & 2775 & Bonn & $=74 / 10.2$ & $-3 \pm 57 / 0.1$ & $601 \pm 49 / 12.3$ & $-32 \pm 42 / 0.8$ \\
\hline HD $164492 C$ & 57099.3 & 2666 & dam & $-495 \pm 92 / 5.4$ & $-95 \pm 89 / 1.1$ & $-544 \pm 66 / 8.2$ & $-31 \pm 62 / 0.5$ \\
\hline & $8 / 13$ & 2612 & Bonn & $=88 / 6.5$ & $-81 \pm 78 / 1.0$ & $-551 \pm 56 / 9.8$ & $-35 \pm 51 / 0.7$ \\
\hline HD 164492B & 56810 & 1530 & lam & $150 / 0.2$ & $273 \pm 177 / 1.5$ & $-44 \pm 130 / 0.3$ & $-199 \pm 110 / 1.8$ \\
\hline & & 1547 & & $138 / 0.4$ & $4 \pm 133 / 0.0$ & $26 \pm 115 / 0.2$ & $-2 \pm 119 / 0.0$ \\
\hline HD 164704 & 56810 & 2667 & & $3 / 0.3$ & $4 / 0.6$ & 46 / 0.4 & $44 / 0.1$ \\
\hline & & & & $1 / 0.7$ & $5 / 1.8$ & $38 / 0.8$ & $36 / 1.5$ \\
\hline HD 166033 & 56810 & 2702 & & 4 / 1.0 & $38 / 0.7$ & $-131 \pm 58 / 2.3$ & $53 / 1.9$ \\
\hline & & 55 & & $5 / 0.5$ & $64 / 0.2$ & 44 / 0.1 & $43 / 0.0$ \\
\hline$-12^{\circ} 4982$ & 5681 & 2510 & am & $30 / 1.0$ & $111 / 0.5$ & $271 \pm 52 / 5.2$ & $53 / 0.8$ \\
\hline & & 2113 & & 8 / 1.0 & $34 / 1.8$ & $266 \pm 57 / 4.7$ & $-21 \pm 47 / 0.4$ \\
\hline 39 & 5681 & 1140 & & $53 / 0.4$ & $36 / 0.4$ & $-22 \pm 116 / 0.2$ & $64 \pm 103 / 0.6$ \\
\hline & $8 / 4$ & 1678 & Bor & $9 \pm 132 / 0.1$ & $25 \pm 108 / 0.2$ & $-4 \pm 79 / 0.1$ & $61 \pm 74 / 0.8$ \\
\hline $\mathrm{CD}-$ & 56810. & 2706 & dam & $-105 \pm 53 / 2.0$ & $-53 \pm 63 / 0.8$ & $-106 \pm 35 / 3.0$ & $-52 \pm 41 / 1.3$ \\
\hline & & 2585 & Bonn & $52 / 1.7$ & $46 / 1.8$ & $-77 \pm 36 / 2.1$ & $\pm 35 / 2.3$ \\
\hline HD 164536 & 56810. & 3472 & dam & \pm 77 / 1.4 & $85 / 0.8$ & $56 \pm 63 / 0.9$ & $-48 \pm 72 / 0.7$ \\
\hline & & 3331 & & 68 / 2.8 & $68 / 1.4$ & $53 / 1.3$ & $=53 / 1.5$ \\
\hline HD 164816 & 5681 & 3576 & & 8 / 0.9 & $2 / 0.5$ & $45 / 1.3$ & $44 / 2.2$ \\
\hline & & & & $1 / 0.7$ & $6 / 0.5$ & $35 / 1.1$ & $33 / 2.3$ \\
\hline HD 165052 & 5681 & & & $2 / 0.9$ & $8 / 0.5$ & $59 / 0.5$ & $71 / 0.7$ \\
\hline & & & & $5 / 0.0$ & $9 / 0.6$ & 49 / 1.5 & $49 / 1.6$ \\
\hline HD 164844 & 5681 & 28 & & $5 / 2.3$ & $8 / 0.1$ & $51 / 1.4$ & $59 / 1.1$ \\
\hline & & 28 & & $1 / 3.0$ & $1 / 0.5$ & $41 / 2.1$ & $42 / 0.3$ \\
\hline 95568 & 5681 & 3170 & & 1 / 1.1 & $8 / 1.0$ & 35 / 0.4 & $37 / 1.1$ \\
\hline & & & & 7 / 1.6 & $45 / 1.1$ & $29 / 1.3$ & $29 / 0.6$ \\
\hline 110150134 & 5681 & & & $28 \pm 51 / 0.5$ & $63 / 1.1$ & $-46 \pm 26 / 1.8$ & $25 \pm 31 / 0.8$ \\
\hline & & & & $53 / 0.7$ & $48 / 0.7$ & $9 \pm 32 / 0.3$ & $8 \pm 31 / 0.3$ \\
\hline HD 156292 & 56811.11206 & 3619 & & $-75 \pm 75 / 1.0$ & $63 / 0.5$ & $33 \pm 51 / 0.6$ & $11 \pm 49 / 0.2$ \\
\hline & & & & $-24 \pm 55 / 0.4$ & $47 / 0.9$ & $24 \pm 35 / 0.7$ & $=34 / 0.5$ \\
\hline HD & 56811 & 3619 & & $31 / 1.9$ & $75 / 0.8$ & $-51 \pm 62 / 0.8$ & $=56 / 0.7$ \\
\hline & & & & $2 / 1.5$ & $66 / 0.2$ & $\pm 52 / 0.9$ & $50 / 0.9$ \\
\hline HD 156234 & 5681 & & & 50 / 1.9 & $88 / 0.0$ & $-114 \pm 31 / 3.7$ & $10 \pm 50 / 0.2$ \\
\hline & & & & 2 / 0.9 & $6 / 0.5$ & 43 / 1.4 & $38 / 0.1$ \\
\hline HI & 5709 & & & 5 / 9.7 & $8 / 0.5$ & $45 / 11.7$ & $45 / 1.2$ \\
\hline & & & & / 7.6 & $8 / 0.0$ & $39 / 12.1$ & $35 / 2.0$ \\
\hline HD 56779 & 5709 & & & 2 / 2.0 & $7 / 0.8$ & $33 / 1.8$ & $41 / 0.1$ \\
\hline & & & & $7 / 3.3$ & $2 / 0.1$ & 29 / 2.5 & $25 / 0.2$ \\
\hline HD 75759 & 57099 & & dar & 71 / 1.4 & $5 / 0.8$ & $-103 \pm 58 / 1.8$ & $77 / 0.4$ \\
\hline & & & & $63 / 0.5$ & $57 / 0.9$ & $-1 \pm 45 / 0.0$ & $41 / 1.0$ \\
\hline HD 110432 & 57099.13247 & 4708 & $F C$ & $-55 \pm 48 / 1.1$ & $55 \pm 55 / 1.0$ & $-62 \pm 39 / 1.6$ & $66 \pm 48 / 1.4$ \\
\hline & & & & $544 \pm 200 / 2.7$ & $129 \pm 183 / 0.7$ & $38 \pm 66 / 0.6$ & $39 \pm 58 / 0.7$ \\
\hline HD 120324 & 57099.16026 & 4833 & $\mathrm{P}$ & $9 \pm 40 / 0.2$ & $80 \pm 48 / 1.7$ & $-22 \pm 31 / 0.7$ & $4 \pm 35 / 0.1$ \\
\hline & $14 / 28$ & 4362 & & $47 \pm 44 / 1.1$ & $26 \pm 33 / 0.8$ & $22 \pm 27 / 0.8$ & $7 \pm 23 / 0.3$ \\
\hline HD 120991 & 57099.18338 & 5919 & Potsdam & $-76 \pm 35 / 2.2$ & $-15 \pm 39 / 0.4$ & $-85 \pm 26 / 3.3$ & $-21 \pm 28 / 0.8$ \\
\hline & $18 / 745$ & 5595 & Bonn & $-77 \pm 60 / 1.3$ & $-26 \pm 32 / 0.8$ & $-76 \pm 25 / 3.0$ & $-15 \pm 19 / 0.8$ \\
\hline CPD $-62^{\circ} 2124$ & 57099.21385 & 1820 & Potsdam & $4636 \pm 128 / 36.2$ & $28 \pm 102 / 0.3$ & $4531 \pm 98 / 46.2$ & $9 \pm 76 / 0.1$ \\
\hline & $8 / 4800$ & 1767 & Bonn & $5222 \pm 123 / 42.5$ & $27 \pm 95 / 0.3$ & $4399 \pm 65 / 67.7$ & $-31 \pm 53 / 0.6$ \\
\hline HD 156233 & 57099.28267 & 2222 & Potsdam & $-92 \pm 124 / 0.7$ & $34 \pm 117 / 0.3$ & $-139 \pm 76 / 1.8$ & $19 \pm 79 / 0.2$ \\
\hline & $8 / 1440$ & 2215 & & $-34 \pm 102 / 0.3$ & $86 \pm 92 / 0.9$ & $-50 \pm 68 / 0.7$ & $48 \pm 63 / 0.8$ \\
\hline HD 315032 & 57099.34159 & 3660 & Potsdam & $-48 \pm 78 / 0.6$ & $-22 \pm 85 / 0.3$ & $-50 \pm 51 / 1.0$ & $-37 \pm 59 / 0.6$ \\
\hline & $16 / 3780$ & 3581 & Bonn & $-69 \pm 75 / 0.9$ & $-31 \pm 56 / 0.6$ & $-43 \pm 47 / 0.9$ & $10 \pm 41 / 0.2$ \\
\hline
\end{tabular}

Notes. Names of objects with magnetic field detections are highlighted in bold face. 

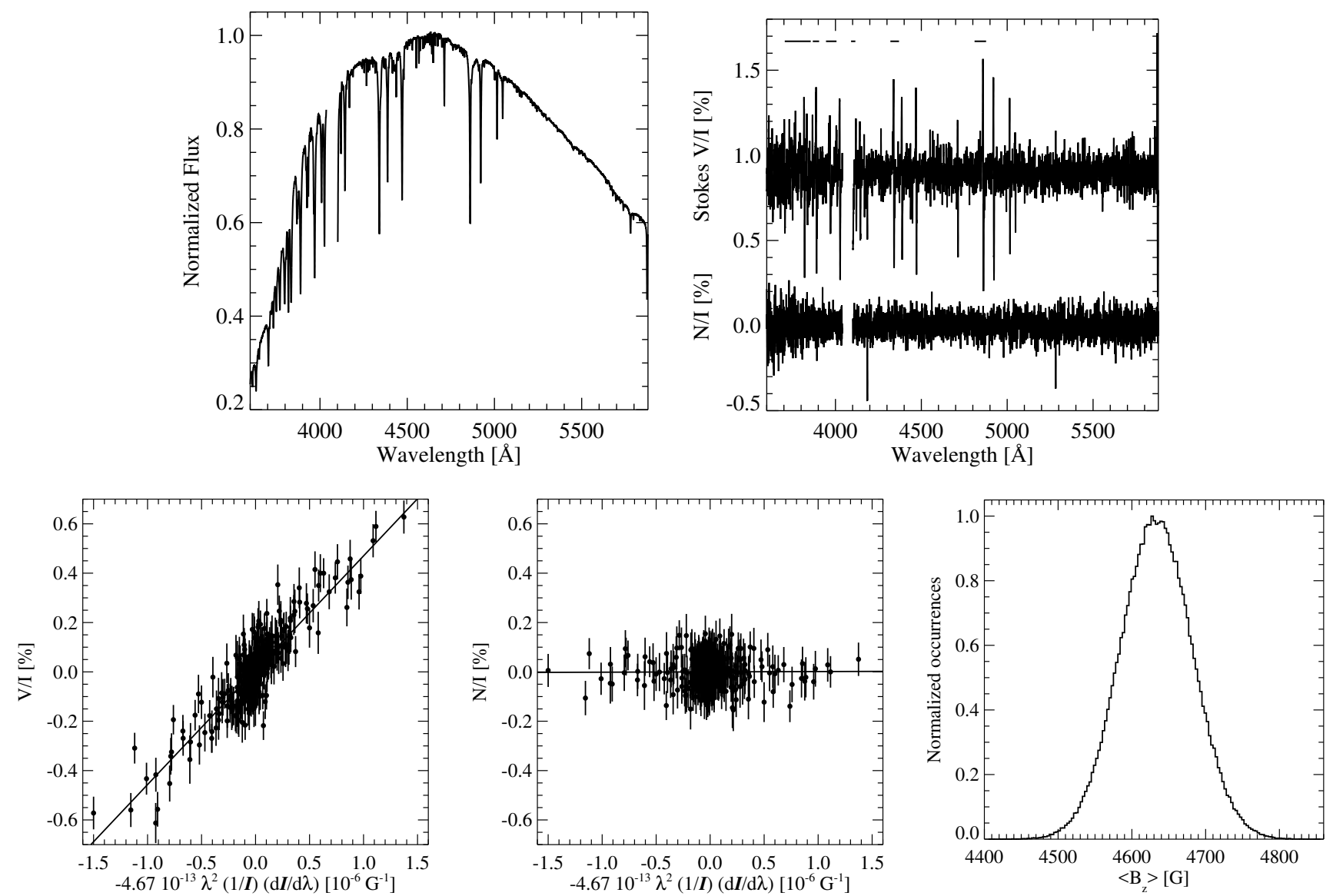

Fig. 1. Overview of the results of the analysis of the FORS 2 data of CPD $-62^{\circ} 2124$, collected during the night of 2015 March 17 , considering the hydrogen lines, using the Potsdam pipeline. Top left panel: observed Stokes I spectrum arbitrarily normalized to the highest value. Top right panel: the top profile shows Stokes $V$ (in \%), while the bottom profile shows the spectrum of the $N$ parameter (in \%). The Stokes $V$ spectrum is shifted upward by $0.9 \%$ for better visibility. The regions used to calculate the longitudinal magnetic field are indicated by horizontal lines close to the top of the panel. Bottom left panel: linear fit to Stokes $V$. Bottom middle panel: linear fit to the $N$ spectrum. From the linear fit, we determine $\left\langle N_{z}\right\rangle=28 \pm 102 \mathrm{G}$. Bottom right panel: distribution of the longitudinal magnetic field values $P\left(\left\langle B_{z}\right\rangle\right)$, which were obtained via bootstrapping. From the distribution $P\left(\left\langle B_{z}\right\rangle\right)$, we obtain the most likely value for the longitudinal magnetic field $\left\langle B_{z}\right\rangle=4636 \pm 128 \mathrm{G}$. The gaps in the region around $\mathrm{H} \delta$ in the two upper panels result from masking an internal reflection in that spectral range.

in Col. 3. The $\mathrm{S} / \mathrm{N}$ is calculated per $\AA$ as the median of the 100 pixels with the highest flux, excluding emission lines. In the next column, we list the reduction software used for the determination of the magnetic field ("Bonn" or "Potsdam"). Columns 5 and 9 show the values for the longitudinal magnetic field determined from the Stokes $V$ spectrum for the hydrogen lines or for the whole spectrum, respectively. Columns 7 and 11 give the same values, but determined from the $N$ spectrum. Columns 6 , 8,10 , and 12 indicate the significance of the respective measurements, defined as the absolute value of the measurement divided by its $1 \sigma$ error. The wavelength ranges used both for the hydrogen lines and for the whole spectrum might differ between the two pipelines. Object names are given in bold face if a magnetic field was found in the measurement.

The detection of strong magnetic fields in the stars HD 54879, CPD $-57^{\circ} 3509$, and HD 164492C was already discussed in the previous paper on BOB FORS 2 observations (Fossati et al. 2015). Individual studies of HD 54879, HD $164492 \mathrm{C}$, and $\mathrm{CPD}-57^{\circ} 3509$ were presented in the papers by Castro et al. (2015), Hubrig et al. (2014), and Przybilla et al. (2016), respectively. We reobserved these objects and find rotational modulation of the magnetic fields in HD 164492C and CPD $-57^{\circ} 3509$, and confirm the magnetic field in HD 54879.

The magnetic nature of the star HD 345439 was already discussed in the work of Hubrig et al. (2015). The analysis of
HD 345439 using four subsequent spectropolarimetric FORS 2 subexposures does not reveal a magnetic field. On the other hand, Hubrig et al. (2015) report that the individual subexposures indicate that HD 345439 may host a strong magnetic field that rapidly varies over $88 \mathrm{~min}$. The fast rotation of HD 345439 is also indicated by the behavior of several metallic and He I lines in the low-resolution FORS 2 spectra that show profile variations already on this short timescale. Wisniewski et al. (2015) found clear evidence that the strength of $\mathrm{H} \alpha$, $\mathrm{He} \mathrm{I}$, and the Brackett series lines indeed vary on a timescale of $\sim 0.7701 \mathrm{~d}$ from their analysis of multiepoch, multiwavelength spectroscopic monitoring.

Apart from these detections, a very strong mean longitudinal magnetic field $\left\langle B_{z}\right\rangle=5222 \pm 123 \mathrm{G}$ (using the Bonn pipeline on the hydrogen lines) in the rather faint $(V=11.0)$ early B-type star CPD $-62^{\circ} 2124$ is discovered in our survey. In Fig. 1, we illustrate the analysis of the hydrogen lines for this object, using the Potsdam pipeline. Such a strong longitudinal magnetic field implies a dipolar magnetic field strength of more than $17 \mathrm{kG}$. Massive stars with such extremely strong magnetic fields are very rare (Bychkov et al. 2009; Petit et al. 2013; Fossati et al. 2015). Follow-up measurements of CPD $-62^{\circ} 2124$ using HARPSpol on one occasion also confirm the presence of an extraordinarily strong magnetic field. A paper presenting an 
individual study of this extremely interesting star was recently submitted by Castro et al. (2017).

Another interesting result is achieved for the star $\mathrm{BD}-12^{\circ} 4982$, where the magnetic field is measured at the $4.7 \sigma$ significance level using the Bonn pipeline and $5.2 \sigma$ using the pipeline in Potsdam. These results are achieved when the whole spectrum is used for the measurements. The measurements using only the hydrogen lines show a significance level below $3 \sigma$. Work on this object using HARPS observations is currently ongoing (Järvinen et al., in prep.).

For a few stars, HD 56779, HD 120991, CD -22 12513 , and HD 164844, longitudinal magnetic fields are detected at a significance level between 3-3.3 $\sigma$, using either both reductions from Bonn and Potsdam or only one of the pipelines. It will be important to monitor these stars with additional FORS 2 observations or to obtain high-resolution spectropolarimetric observations to scrutinize more methodically for the presence of a magnetic field.

It would be especially interesting to carry out a follow-up study of the Be star HD 120991, since there is currently no undisputed evidence of a magnetic field in any classical Be star. Hubrig et al. (2009) carried out a search for magnetic fields in $16 \mathrm{Be}$ stars and concluded that magnetic fields in such stars are usually very weak, i.e., below $100 \mathrm{G}$. Bagnulo et al. (2012, 2015) could not confirm the magnetic field detections from the same data sets. The MiMeS group reported that none of the $85 \mathrm{Be}$ stars studied with ESPaDOnS, Narval, and HARPSpol showed the presence of a magnetic field, with a median $1 \sigma$ error of $103 \mathrm{G}$ (Wade et al. 2014). In our sample of 71 stars, we included four Be stars: HD 110432, HD 120324, HD 120991, and HD 315032. For the first three Be stars, hard X-ray emission was detected using XMM-Newton observations (see Sect. 5.3). We obtain magnetic fields at a 3-3.3 $\sigma$ level only for the Be star HD 120991, using both pipelines applied to the whole spectrum. Should the presence of a magnetic field in HD 120991 be established with additional measurements, it would be weak.

\section{In-depth analysis of the magnetic field measurements}

\subsection{Comparison of the two data reduction pipelines}

The FORS 2 spectropolarimetric data obtained within the BOB Collaboration was independently reduced and analyzed by two teams using different tools and pipelines. This gives us the possibility to directly compare the results for a statistically large sample of stars.

Figure 2 shows the comparison between the results obtained by reducing and analyzing the spectra with the Bonn and Potsdam pipelines. We consider here all 134 sets of measurements (this work and Fossati et al. 2015), in which each set is composed of two measurements (i.e., $\left\langle B_{z}\right\rangle$ obtained from the analysis of the hydrogen lines or of the whole spectrum), and obtained by the two pipelines, for a total of 536 measurements. As was already evident from the analysis of a subset of the data we present in Fig. 2 (see Fossati et al. 2015), there is generally good agreement between the reduction and analysis of the two groups, with the occasional outlier.

To allow a better quantification of the agreement between the results of the two groups, we compared the difference between the two distributions of magnetic field measurements from Bonn $(\mathrm{BN})$ and Potsdam (P) with a Gaussian distribution. For this, we computed for each of the 134 measurements the following value:

$$
\left\langle B_{z}\right\rangle_{\mathrm{diff}}=\frac{\left\langle B_{z}\right\rangle_{\mathrm{BN}}-\left\langle B_{z}\right\rangle_{\mathrm{P}}}{\sqrt{\sigma_{\left\langle B_{z}\right\rangle, \mathrm{BN}}^{2}+\sigma_{\left\langle B_{z}\right\rangle, \mathrm{P}}^{2}}},
$$

which is the error-normalized difference between the $\left\langle B_{z}\right\rangle$ values measured by both the Bonn and Potsdam groups. The normalization was made using the square root of the sum of the squared errors coming from the Bonn and Potsdam analyses, following error propagation.

In Fig. 3, we show the resulting density distributions for the differences from the measurements of the hydrogen lines (top) and from the whole spectrum (bottom). In these graphs, all values calculated according to Eq. (2) are sorted and then plotted corresponding to their rank in the distribution. The solid overlayed line shows the density distribution for a Gaussian. While the density distribution for the normalized differences for the $\left\langle B_{z}\right\rangle$ obtained from all lines corresponds well with a Gaussian distribution, the equivalent density distribution for the hydrogen lines is much narrower, owing to the larger errors. We would like to stress that strictly speaking it is only possible to make statistical comparisons in the way presented here for repeated measurements of the same value, but not for individual measurements of different values. Also, the difference of two similar Gaussian distributions results in a distribution that is a factor of $\sqrt{2}$ wider than the original distributions, i.e., the distribution coming from the difference of our two measurements is narrower than should be expected, pointing to a correlation between the Bonn and Potsdam reduction and analysis approaches.

One measurement is not shown for the hydrogen density distribution, since the difference between the measurements is very large. This outlier is CPD $-62^{\circ} 2124$, where we have a normalized difference of 3.3. This is due to the $12 \%$ difference in the value for the magnetic field determined by both groups and the high significance of the result (see also Fossati et al. 2015).

Overall, our results show that differences between the two data reduction and analysis pipelines are usually well within $3 \sigma$. The differences become important when evaluating measurements near the $3 \sigma$ threshold and when assessing the absolute values of the magnetic fields measured with high significance.

\subsection{Signal-to-noise ratio in spectra and resulting errors}

In Fig. 4, we show the dependence of the derived errors of the magnetic field measurements on the $\mathrm{S} / \mathrm{N}$ obtained in the raw spectra. It is obvious that with higher $\mathrm{S} / \mathrm{N}$, i.e., higher flux, the errors become smaller. Using the whole spectrum (circles) results in smaller errors than using only the hydrogen lines (squares). Also, the Potsdam reduction usually delivers spectra with a slightly higher $\mathrm{S} / \mathrm{N}$ than the Bonn reduction. The errors derived from the Bonn analysis are usually slightly smaller than from the Potsdam analysis, mainly due to accounting for deviations from the nominal CCD gain via $\chi^{2}$ scaling (see Fossati et al. 2015). The outlier from the Bonn pipeline using only the hydrogen lines is from HD 110432, where the Bonn pipeline excludes the various emission lines.

The measurement error $\sigma$ depends not only on the $\mathrm{S} / \mathrm{N}$, but also on the nature of the spectrum, i.e., spectral type, $v \sin i$, binarity, etc. For the same $\mathrm{S} / \mathrm{N}, \sigma$ is expected to be lower for observations of early B-type stars compared to observations of O-type stars. The FORS 2 magnetic field detections in O-type stars that have been confirmed with high-resolution spectropolarimetry rarely reach $4 \sigma$. Based on our data, where only very 

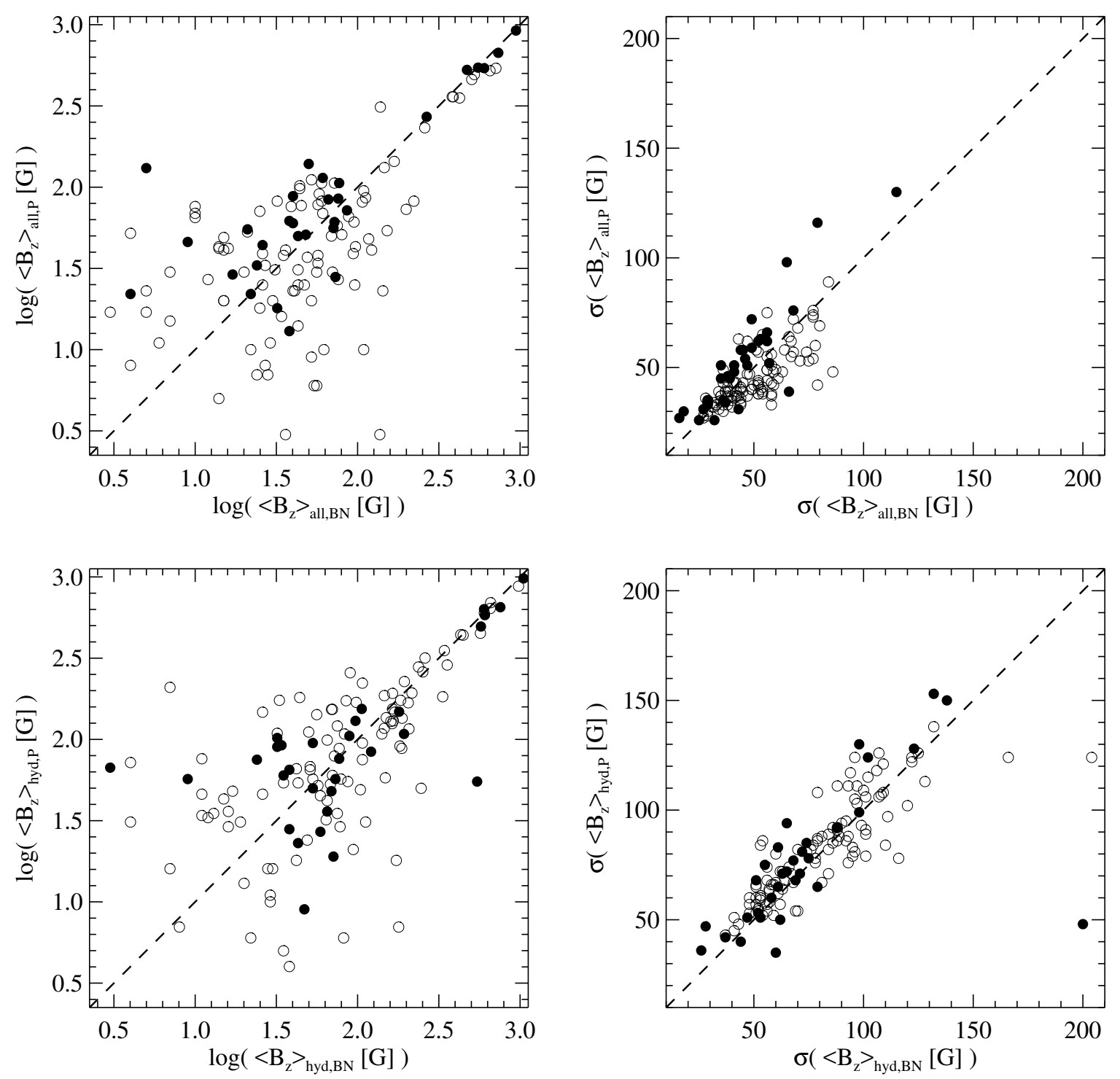

Fig. 2. Top left panel: comparison between the $\left|\left\langle B_{z}\right\rangle\right|$ values obtained by analyzing the whole spectrum with the Bonn pipeline and the Potsdam pipeline. Top right panel: same as top left panel, but for the uncertainties of the $\left\langle B_{z}\right\rangle$ values obtained analyzing the whole spectrum. Bottom left panel: same as top left panel, but for the $\left\langle\left\langle B_{z}\right\rangle\right|$ values obtained analyzing only the hydrogen lines. Bottom right panel: same as top left panel, but for the uncertainties of the $\left\langle B_{z}\right\rangle$ values obtained analyzing only the hydrogen lines. Filled circles are from measurements presented in this paper, while open circles are from the measurements presented in Fossati et al. (2015). The extreme outlier in the bottom right panel in the lower right is from the Be star HD 110432 (see the discussion in the text).

few stars were studied at a $\mathrm{S} / \mathrm{N}$ higher than 4000 , we cannot make conclusions about the saturation of $\sigma$ at even higher $\mathrm{S} / \mathrm{N}$.

Bagnulo et al. (2015) presented a comparable analysis (see their Fig. 5) for $\sim 1400$ data sets obtained with FORS 1 in spectropolarimetric mode, which mainly concentrated on the errors obtained from the analysis of the null spectra. While their results are qualitatively similar, it can be seen that our data typically have higher $\mathrm{S} / \mathrm{N}$ than the heterogeneous FORS 1 sample.

\subsection{Rectification}

All Stokes $V$ spectra were rectified to ensure that the continuum is consistent with zero (see as an example the work by Hubrig et al. 2014 and Fossati et al. 2015). The offsets of the non-rectified Stokes $V$ spectra from 0 are typically small, below 0.0015 . Rectification usually leads to a vertical shift of the spectra. Among the whole sample of 71 stars discussed in this paper and in Fossati et al. (2015), the non-rectified $V$ spectrum is tilted only for the Be star HD 110432 and the spectroscopic binary HD 92206C, with an O6.5V((f)) primary. Such a behavior of the Stokes $V$ spectrum is sometimes observed in Herbig Ae/Be stars because of the presence of a circumstellar disk (Hubrig et al., in prep.). A variable tilt of the Stokes $V$ spectrum over the orbital phase was detected in the Cyg X-1 system, which consists of an O-type supergiant and a black hole (Karitskaya et al. 2010). Usually, this type of feature is attributed to cross talk between linear and circular polarization and is seen in sources that show strong linear polarization (e.g., Bochkarev \& Karitskaya 2012; Bagnulo et al. 2012).

In Fig. 5, we present the rectified and non-rectified spectra of HD 110432 and for the three observations of HD 92206C. While the rectification has only some influence on the determination of the magnetic field in HD 110432, leading to a value of $\left\langle B_{z}\right\rangle=$ $22 \pm 46 \mathrm{G}$ for the magnetic field from the non-rectified spectrum 

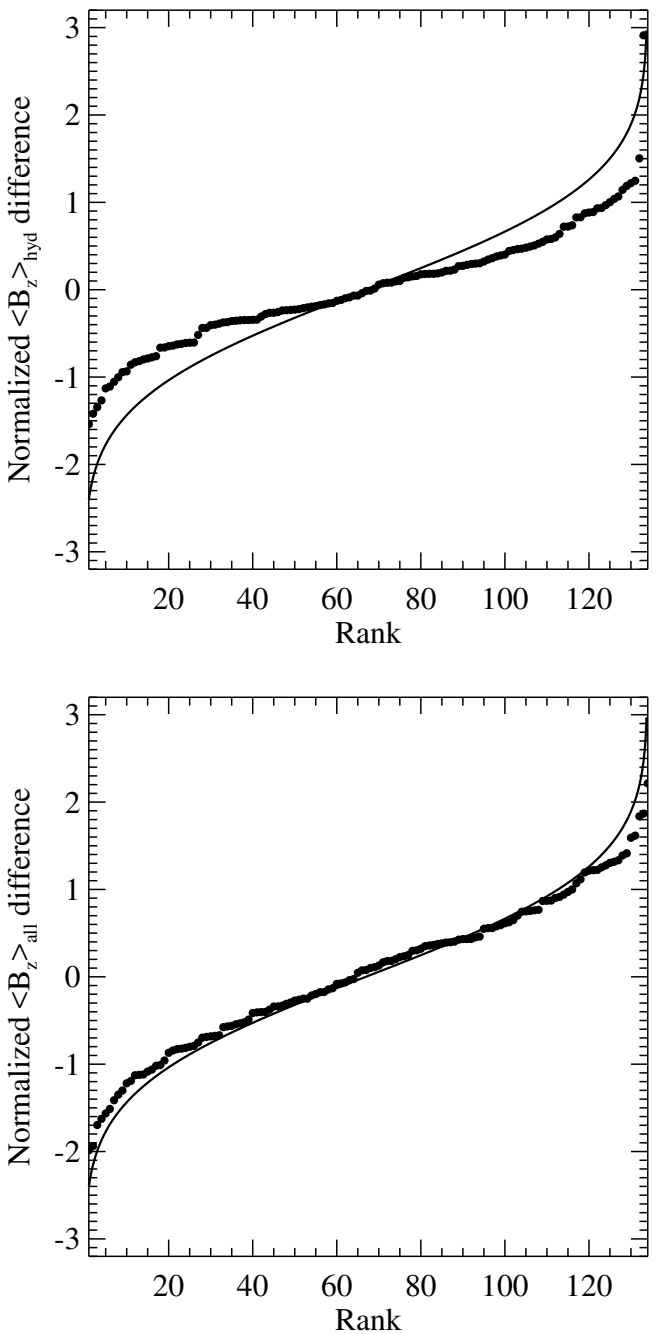

Fig. 3. Density distributions for the error-normalized differences between the magnetic field values determined by the Bonn and Potsdam groups for the 134 measurements presented in Fossati et al. (2015) and in this paper. The overlayed lines are for a Gaussian density distribution. Top panel: normalized differences for the $\left\langle B_{z}\right\rangle$ obtained from hydrogen lines. Bottom panel: normalized differences for the $\left\langle B_{z}\right\rangle$ obtained from all lines.

compared to $\left\langle B_{z}\right\rangle=-62 \pm 39 \mathrm{G}$ from the rectified spectrum, the influence is significant for HD 92206C. For this object we obtain values for the magnetic field of $\left\langle B_{z}\right\rangle=366 \pm 103 \mathrm{G},\left\langle B_{z}\right\rangle=208 \pm$ $113 \mathrm{G}$, and $\left\langle B_{z}\right\rangle=239 \pm 94 \mathrm{G}$, respectively, for the three nights from the non-rectified spectra, compared to $\left\langle B_{z}\right\rangle=76 \pm 64 \mathrm{G}$, $\left\langle B_{z}\right\rangle=-48 \pm 72 \mathrm{G}$, and $\left\langle B_{z}\right\rangle=-39 \pm 55 \mathrm{G}$ from the rectified spectra. All values were determined using the whole spectrum and the Potsdam pipeline, which employs a linear fit to rectify the $V$ spectrum.

The Bonn pipeline, which uses a fourth-order polynomial for the rectification, leads to similar results. Without rectification, the measurements of HD 92206C give $\left\langle B_{z}\right\rangle=357 \pm 98 \mathrm{G},\left\langle B_{z}\right\rangle=$ $209 \pm 104 \mathrm{G}$, and $\left\langle B_{z}\right\rangle=313 \pm 97 \mathrm{G}$, respectively, while the rectified spectra lead to $\left\langle B_{z}\right\rangle=39 \pm 66 \mathrm{G},\left\langle B_{z}\right\rangle=-117 \pm 68 \mathrm{G}$, and $\left\langle B_{z}\right\rangle=-26 \pm 56 \mathrm{G}$.

\subsection{Spectral variations from pulsations}

A number of stars in our sample of 71 objects (this paper and Fossati et al. 2015) observed with FORS 2 are reported

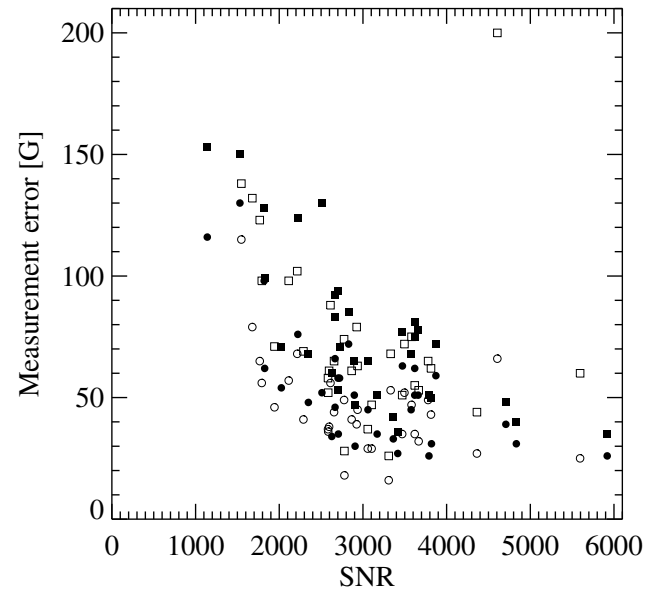

Fig. 4. Derived $1 \sigma$ errors plotted against the obtained $\mathrm{S} / \mathrm{N}$ for the magnetic field measurements presented in Table 2. Circles denote measurements using the whole spectrum, squares indicate measurements using only the hydrogen lines, filled symbols indicate results from the Potsdam reduction and analysis, and open symbols denote results from the Bonn reduction and analysis.
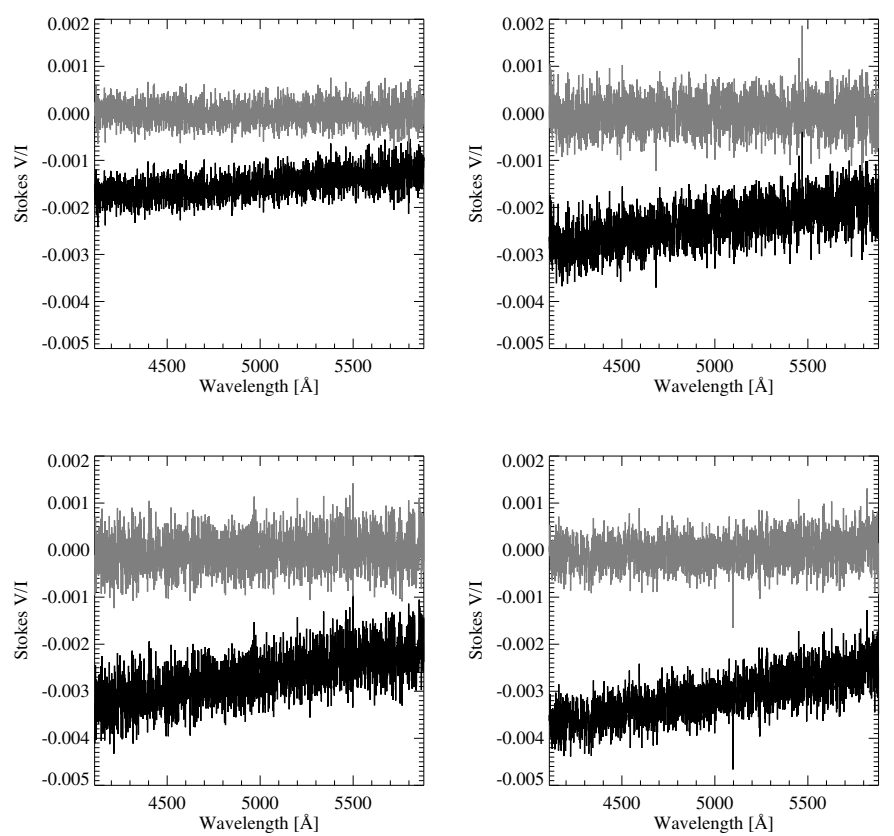

Fig. 5. Non-rectified (black) and rectified (gray) Stokes $V / I$ spectra of the Be star HD 110432 (top left) and the spectroscopic binary HD 92206C with an $\mathrm{O} 6.5 \mathrm{~V}((\mathrm{f}))$ primary on three consecutive nights, determined using the whole spectrum and the Potsdam pipeline.

in the literature to exhibit different types of pulsations. From high-resolution spectropolarimetric observations, pulsations are known to have an impact on the analysis of the presence of a magnetic field and its strength (e.g., Schnerr et al. 2006; Hubrig et al. 2011; Neiner et al. 2012). Sometimes, it is also possible to observe features that are similar to Zeeman patterns expected in Stokes $V$ spectra in null spectra. This depends on the pulsation phase, i.e., on the radial velocity amplitude and profile shape changes, and on the ratio between the exposure time and pulsation period. These features can be seen in observations where the duration of the subexposures taken at different retarder waveplate angles is a significant portion of the pulsation cycle. Unfortunately, so far the effect of pulsations on the low-resolution 
spectropolarimetric observations with FORS 2 has not been thoroughly investigated.

The following objects from the full sample of 71 stars are known to show pulsations.

- HD 44597: This is a candidate short-period variable observed by HIPPARCos and found by Koen \& Eyer (2002). No frequency was found by Pigulski \& Pojmanski (2008). Buysschaert et al. (2015) did not find a clear periodic variability with isolated frequencies from Kepler data, but several low-frequency peaks stand out from the noise level. The current speculative interpretation are convectively driven internal gravity waves (e.g., Rogers et al. 2013).

- HD 46149: Degroote et al. (2010) detected rotational modulation and stochastic $p$ modes from 3.05 to $7.23 \mathrm{~d}^{-1}$ from CoRoT data.

- HD 46150 and HD 46966: Blomme et al. (2011) detected in both stars a red noise power excess in CoRoT data, which was interpreted as convectively driven internal gravity waves of a stochastic nature by Aerts \& Rogers (2015).

- HD 46202: Briquet et al. (2011) used CoRoT data to detect heat-driven $p$ modes, similar to $\beta$ Cephei-like modes with pulsation frequencies of $0.5-4.9 \mathrm{~d}^{-1}$.

- HD 46223: Blomme et al. (2011) detected a red noise power excess in CoRoT data, which was interpreted as convectively driven internal gravity waves of a stochastic nature by Aerts \& Rogers (2015). The detected frequencies were 2.007, 4.011, and $13.792 \mathrm{~d}^{-1}$.

- HD 46328: This is a well-known $\beta$ Cephei star with a period of $\sim 5 \mathrm{~h}$ (Saesen et al. 2006, and references therein).

- HD 64365: Telting et al. (2006) found $\beta$ Cephei pulsations of low degree.

- HD 93521: Howarth \& Reid (1993) and Rauw et al. (2012) found that the stellar pulsations in this star display a biperiodic (1.75 and $2.89 \mathrm{~h}$ ) absorption line profile variability that is commonly interpreted as the signature of two nonradial pulsation modes.

- HD 95568: Pigulski \& Pojmanski (2008) found $\beta$ Cephei pulsations with a frequency of $6.152355 \mathrm{~d}^{-1}$.

- HD 117357: Pigulski \& Pojmanski (2008) found $\beta$ Cephei pulsations with frequencies of $2.08106(3) \mathrm{d}^{-1}$ and $6.53990 \mathrm{~d}^{-1}$.

- HD 144470: Telting et al. (2006) found high degree $\beta$ Cephei pulsations.

Not all stars that exhibit pulsations also showed changes between the spectra of the different subexposures. HD 46328, HD 64365 , and HD 95568 were all observed three times, and HD 117357 was observed twice. All spectra of these four stars showed profile changes within the observing sequence. The single data set for HD 46966 also showed small changes in the spectra. HD 44597 was observed on two nights. While profiles changed during the first night, no variability was found on the second night. HD 46202 was observed three times. Profile changes can be seen in the first two data sets, but there are no changes in the last one. No variations were found in the spectra of HD 46149, HD 46150, and HD 46223, which were each observed during a single epoch, during the six epochs for HD 144470, or the three epochs for HD 93521.

It is possible that pulsations are a cause for spurious magnetic field detections. However, they might also be traceable as significant values of the diagnostic $\left\langle N_{z}\right\rangle$. Looking at the $\left\langle N_{z}\right\rangle$ measurements in the full data set of 71 stars, in the following five objects $\left\langle N_{z}\right\rangle$ exceeds $3 \sigma$ in at least one of the measurements: HD 46328, HD 95568, HD 101008, HD 102475 (twice), and HD 289002. While the first two objects are known pulsators (see above), nothing is known about pulsations in the other three stars. They all have a spectral type of B1 and might be pulsation candidates.

\section{Exploring indirect magnetic field indicators}

Two properties were suggested in the past as indirect magnetic field indicators: slow rotation and X-ray characteristics. Furthermore, as was already reported in previous studies, the occurrence of magnetic stars with radiative envelopes in close binary or multiple systems is extremely low (e.g., Carrier et al. 2002; Schöller et al. 2012; Grunhut \& Wade 2013). This motivated Ferrario et al. (2009) to suggest a merger scenario to explain the origin of magnetic fields. According to this scenario, if mergers were the sole mechanism to produce a magnetic field in an OB star, no magnetic fields would be expected in a close binary. To investigate the usefulness of these indicators, we studied all 71 stars in our sample observed with FORS 2. About one-third of our targets were selected on the basis of their low $v \sin i$, a few were explicitly selected for their hard X-rays. In the following we discuss these properties for our FORS2 sample.

\subsection{Rotation}

Morel et al. (2008) suggested that magnetic OB stars are mostly slowly rotating and nitrogen rich. Following their arguments, we selected 20 stars that have a rotational velocity $v \sin i$ below $60 \mathrm{~km} \mathrm{~s}^{-1}$ from the catalogs of Howarth et al. (1997) and Simón-Díaz \& Herrero (2014).

From these 20 stars, only HD 54879 shows a magnetic field. The other three targets with detected magnetic fields were not selected on the basis of their low $v \sin i$. In the subsequent studies by Przybilla et al. (2016) on CPD $-57^{\circ} 3509$ and Castro et al. (2017) on CPD $-62^{\circ} 2124$, both stars were found to have a $v \sin i$ of $35 \mathrm{~km} \mathrm{~s}^{-1}$. HD 164492A was selected on the basis of its low $v \sin i$, but a magnetic field was found in the component HD 164492C (Hubrig et al. 2014; González et al. 2017).

\subsection{Multiplicity}

In the following, we list known companions to the systems in our sample. We limited this compilation to companions closer than $3^{\prime \prime}$, which corresponds to the worst seeing conditions under which we observed.

- $C P D-59^{\circ}$ 2624: The Washington Double Star (WDS) Catalog (Mason et al. 2001) lists a companion at a distance of 0 '. 1 with a visual magnitude of 12.3 .

- HD 37020: This is $\theta^{1}$ Ori A, which consists of two visual components. The WDS Catalog gives a distance of 0 !'2 for the pair. Pourbaix et al. (2004) list one of the two components as a spectroscopic binary. Its period is under discussion with values of 65.4 or $6.5 \mathrm{~d}$.

- HD 46150: The WDS Catalog lists companions at 2'.1 and $3^{\prime \prime}$ with visual magnitudes of 13.65 and 12.4 , respectively,

- HD 46202: Sana et al. (2014) resolved a companion with the Sparse Aperture Mask mode (SAM; Tuthill et al. 2010) of NAOS-CONICA (Lenzen et al. 2003; Rousset et al. 2003) at a distance of 85.5 mas.

- HD 46223: The WDS Catalog lists a companion at a distance of $0 . .5$ with a visual magnitude of 12 .

- HD 46966: The WDS Catalog lists one companion at a distance of $0 \prime \prime 1$ with a visual magnitude of 8.3. Sana et al. (2014) detected this companion with SAM at a distance of 50.5 mas. 
- HD 72648: The WDS Catalog lists one companion at a distance of 1 !'7 with a visual magnitude of 14.2.

- HD 72754: Pourbaix et al. (2004) list this system as a spectroscopic binary with a period of $33.734 \mathrm{~d}$.

- HD 75759: Sota et al. (2014) list HD 75759 as a doublelined spectroscopic binary. Pourbaix et al. (2004) give a period of $33.311 \mathrm{~d}$ and an orbital eccentricity of 0.63. Sana et al. (2014) resolved this system both in 2012 and 2013 with PIONIER (Le Bouquin et al. 2011) on the Very Large Telescope Interferometer (e.g., Schöller 2007). They found a sub-milliarcsecond companion, whose exact position and magnitude in the $H$ band were determined with very large error bars.

- HD 92206C: Sana et al. (2014) resolved this system with SAM and found a companion at a distance of about 30 mas. Sota et al. (2014) list HD 92206C as a double-lined spectroscopic binary.

- HD 152218: Pourbaix et al. (2004) list this object as a spectroscopic binary, with a period of $5.4 \mathrm{~d}$.

- HD 152246: Sana et al. (2014) resolved this system with PIONIER and found a companion at a distance of 3 mas. Sota et al. (2014) list HD 152246 as a double-lined spectroscopic binary.

- HD 152590: Pourbaix et al. (2004) list this object as a spectroscopic binary, with a period of $4.5 \mathrm{~d}$.

- HD 156292: This object is a double-lined spectroscopic binary with a period of $4.94 \mathrm{~d}$ (Sota et al. 2014).

- HD 164492A: Sana et al. (2014) resolved this system with both PIONIER and SAM and found a companion at a distance of between 25 and 33 mas. The WDS Catalog lists another companion at a distance of 1 ".5 with a visual magnitude of 13. Sota et al. (2014) list HD 164492A as a potential single-lined spectroscopic binary.

- HD 164492C: Hubrig et al. (2014) showed that at least two components are visible in the HARPS spectra of this magnetic star. Further analysis confirmed the presence of three components in the spectra (González et al. 2017). According to the WDS Catalog, component HD 164492D is at a distance of between $2, ! 3$ and 2 ".9.

- HD 164536: The WDS Catalog lists a companion at distances between 1'.5 and 1'!7 with a visual magnitude of 12.4.

- HD 164816: This system was resolved by Sana et al. (2014) both with PIONIER and SAM in 2012. PIONIER found the companion at a distance of 57 mas with a magnitude difference of 3.5 in the $H$ band. Sota et al. (2014) list HD 164816 as a double-lined spectroscopic binary.

- HD 165052: Morrison \& Conti (1978) published the first orbit for this spectroscopic binary with a period of $6.14 \mathrm{~d}$, which was later revised by Stickland et al. (1997) as $2.96 \mathrm{~d}$.

- HD 168625: The WDS Catalog lists one companion at a distance of 1 !' 1 with a visual magnitude of 12.6 .

In total, 16 out of the 71 objects in our sample have known companions at a distance below $1^{\prime \prime}$. Among these multiple systems, a magnetic field at high confidence level was only found in the star HD 164492C.

\subsection{X-ray properties}

Although strong and hard X-ray emission is often used as an indirect indicator of the presence of a magnetic field in massive stars (Ignace et al. 2013; Nazé et al. 2014; ud-Doula \& Nazé 2016), previous studies have demonstrated that this condition is not a necessary indicator of stellar magnetism, and that magnetic stars can be insignificant X-ray sources (Oskinova et al. 2011; Nazé et al. 2014). Using the FORS 2 observations, we intended to check whether strong X-ray emission always results from a magnetic field and thus may be a good magnetic field indicator.

For this purpose, we extensively searched X-ray archives and selected the following early B-type stars with unusual X-ray characteristics: HD 166033, HD 110432, HD 120991, and HD 120324 (see Table 3). A detailed discussion of the X-ray properties of these stars will be presented in a forthcoming publication (Oskinova et al., in prep.). We also obtained X-ray observations of the known magnetic B-type star HD 345439 with the $X M M-N e w t o n$ telescope. Below, we briefly discuss the results of our search for a correlation between X-rays and magnetic properties for the objects described in this study.

HD 166033 is thought to be the main ionizing source of the nebula around IC 1274 (Dahm et al. 2012). The high X-ray luminosity of this B1V star is outstanding (however, one has to keep in mind the possible uncertainty on the distance). For example, the X-ray luminosity of the well-known magnetic stars $\tau$ Sco (B0.2V) and $\xi^{1}$ CMa (B0.7IV) are two orders of magnitude lower (Oskinova et al. 2011, 2014). Yet, no magnetic field was detected in HD 166033.

Among our targets there is also HD 110432, which is a hard and bright X-ray source. This Be star is rotating near critical velocity, and is classified as a $\gamma$ Cas-analog (e.g., Motch et al. 2015, and references therein). It was suggested that a magnetic field may be responsible for the unusual X-ray properties of these enigmatic objects (Smith et al. 2016). However, our measurements do not confirm the presence of a strong magnetic field in this object. Here, we report that its spectroscopic twin, HD 120991, has similar X-ray properties, and propose HD 120991 as a $\gamma$ Cas-analog. Similarly, the X-ray luminosity of another of our targets, HD 315032, is comparable to that of the $\gamma$ Cas-analogs, and its low signal-to-noise X-ray spectrum indicates that this star is a hard X-ray source as well. Further studies are needed to confirm it as a $\gamma$ Cas-analog. No strong magnetic field was detected in these objects either.

The X-ray properties of the $\gamma$ Cas-analogs are in sharp contrast with those of HD 120324, which is another fast rotating Be star. Its X-ray luminosity is low and the spectrum is very soft. Our study allows us to rule out a strong stellar magnetic field as a reason for this discrepancy.

The fast rotating star HD 345439 is an analog of $\sigma$ OriE, but its X-ray luminosity is about two orders of magnitude lower. This object is comparable to that of the magnetic B stars V1046 Ori, $\zeta$ Cas, V2052 Ori, and LP Ori. It was shown in the past that magnetic early-type stars can be insignificant X-ray sources (e.g., Oskinova et al. 2011; Ignace et al. 2013). We should note that $\sigma$ Ori $\mathrm{E}$ almost certainly has a low-mass active companion (e.g., Sanz-Forcada et al. 2004; Bouy et al. 2009).

$\mathrm{BD}-12^{\circ} 4982$ is a likely member of the NGC 6604 cluster and one of its brightest X-ray sources. To estimate its X-ray luminosity, we adopted the cluster distance and reddening from Kharchenko et al. (2009). It appears that this object has unremarkable X-ray properties. A magnetic field in BD $-12^{\circ} 4982$ was measured in the whole spectrum at the $4.7 \sigma$ and $5.2 \sigma$ levels.

The X-ray emission from HD 164816 was studied in detail by Trepl et al. (2012), who detected X-ray pulsations with a period of $9.78 \mathrm{~s}$ in this object. The faint, close companion to HD 164816 found by Sana et al. (2014) could be an active premain-sequence star responsible for the X-ray emission (see also Sect. 5.2). No magnetic field was detected in this source.

HD 165052 is another massive binary. Presumably, the bulk of its X-ray emission is produced in the colliding winds of the 
Table 3. Objects in our FORS 2 sample with available X-ray observations.

\begin{tabular}{|c|c|c|c|c|c|}
\hline Object & $\begin{array}{l}\text { Spectral } \\
\text { type }\end{array}$ & $\begin{array}{c}L_{\mathrm{X}} \\
{\left[10^{31} \mathrm{erg} \mathrm{s}^{-1}\right]}\end{array}$ & $\begin{array}{c}\text { Adopted } \\
\text { distance } \\
{[\mathrm{kpc}]}\end{array}$ & References & Comments \\
\hline $\mathrm{BD}-12^{\circ} 4982$ & B0 II & 1 & 1.5 & 1 & \\
\hline HD 110432 & B2 pe & 40 & 0.37 & 2,3 & $\gamma$ Cas-analog \\
\hline HD 120324 & B2 Vnpe & 0.06 & 0.115 & 4 & \\
\hline HD 120991 & B2 IIne & 30 & 0.83 & 4,5 & $\gamma$ Cas-analog? \\
\hline HD 164816 & $09.5 \mathrm{~V}+\mathrm{B} 0 \mathrm{~V}$ & 3 & 6 & 6 & X-ray pulsations \\
\hline HD 165052 & $07 \mathrm{Vz}+07.5 \mathrm{Vz}$ & 300 & 1.2 & 7 & colliding wind binary \\
\hline HD 166033 & $\mathrm{~B} 1 \mathrm{~V}$ & 800 & 1.5 & 4,8 & \\
\hline HD 315032 & B2 Vne & 10 & 1.2 & 9 & \\
\hline HD 345439 & $\mathrm{~B} 1 / \mathrm{B} 2 \mathrm{~V}$ & $<0.05$ & 1.5 & 10 & $\sigma$ Ori E-analog \\
\hline CPD $-59^{\circ} 2624$ & $09.5 \mathrm{~V}$ & 1.9 & 2.3 & 11 & \\
\hline HD 37020 & B $0.5 \mathrm{~V}$ & 25 & 0.45 & 12 & \\
\hline HD 46056 & $\mathrm{O} 8 \mathrm{~V}$ & 32.5 & 1.4 & 13 & \\
\hline HD 46106 & O9.7 III & 0.9 & 1.4 & 13 & \\
\hline HD 46149 & $\mathrm{O} 8.5 \mathrm{~V}((\mathrm{f}))$ & 1.1 & 1.4 & 13 & \\
\hline HD 46150 & $\mathrm{O} 5 \mathrm{~V}((\mathrm{f}))$ & 22 & 1.4 & 13 & \\
\hline HD 46202 & O9 V((f)) & 1.3 & 1.4 & 13 & \\
\hline HD 46223 & $\mathrm{O} 4 \mathrm{~V}((\mathrm{f}))$ & 24 & 1.4 & 13 & \\
\hline HD 46328 & B0.7 IV & 3 & 0.42 & 14 & X-ray pulsations \\
\hline HD 60848 & O8:V: & 0.13 & 0.5 & 15 & \\
\hline HD 93027 & O9.5 IV & 1.3 & 2.3 & 11 & \\
\hline HD 93521 & O9 Vp & 1.1 & 1.2 & 16 & \\
\hline HD 101008 & B1 II/III & 3 & 2.3 & 17 & \\
\hline HD 117357 & $09.5 / \mathrm{B} 0 \mathrm{~V}$ & 20 & 7 & 18 & \\
\hline HD 125823 & B7 IIIpv & 0.005 & 0.14 & 19 & \\
\hline HD 152246 & O9 IV & 14 & 1.6 & 20 & triple \\
\hline
\end{tabular}

Notes. Distances and the interstellar absorption column density - used to derive $L_{\mathrm{X}}$ - are estimated on the basis of $U B V$ photometry, or taken from the literature.

References. (1) De Becker et al. (2005); (2) Motch et al. (2015); (3) Torrejón et al. (2012); (4) Oskinova et al. (in prep.); (5) Frémat et al. (2002); (6) Trepl et al. (2012); (7) Pittard \& Parkin (2010); (8) Dahm et al. (2012); (9) Damiani et al. (2004); (10) Eikenberry et al. (2014); (11) Nazé et al. (2011); (12) Stelzer et al. (2005); (13) Wang et al. (2008); (14) Oskinova et al. (2014); (15) Rauw et al. (2013); (16) Rauw et al. (2012); (17) Nazé et al. (2013); (18) Beer (1961); (19) Nazé et al. (2014); (20) Nasseri et al. (2014).

two O stars (Pittard \& Parkin 2010). We did not detect a magnetic field in this source.

An overview about the known X-ray properties of the 71 objects in our survey is given in Table 3. In Cols. 1 and 2, we list the object name and the spectral type, followed by the X-ray luminosity in Col. 3, and the adopted distance in Col. 4. Column 5 gives the corresponding reference and the last column provides comments on the object. The upper part of the table lists objects studied in this article, and the lower part lists objects reported in Fossati et al. (2015). No details on X-rays are reported for the sources not listed in Table 3. However, even this limited study confirms previous reports that while some magnetic stars are strong X-ray sources, others are not. Moreover, strong and hard X-rays from presumably single massive stars are not necessarily associated with the presence of stellar magnetic fields, and hence cannot serve as their indirect indicators (e.g., Petit et al. 2013).

\section{Discussion and conclusions}

The $B$ fields in OB stars Collaboration aims at characterizing the incidence of magnetic fields in slowly rotating massive stars. As part of this study, we employed the low-resolution FORS 2 spectropolarimeter to observe a total of $71 \mathrm{OB}$ stars, focusing on the fainter, less studied, objects and the faster rotators in our sample.
From the 32 observations of $28 \mathrm{OB}$ stars reported in this article, we were able to monitor the magnetic fields in CPD $-57^{\circ} 3509$ (Przybilla et al. 2016) and HD 164492C (Hubrig et al. 2014), confirm the magnetic field in HD 54879 (Castro et al. 2015), and detect a magnetic field in CPD -62 2124 (Castro et al. 2017). While the standard analysis of HD 345439 did not reveal a magnetic field, the individual subexposures indicate that HD 345439 may host a strong magnetic field that rapidly varies over $88 \mathrm{~min}$ (Hubrig et al. 2015). A magnetic field in BD $-12^{\circ} 4982$ was measured in the whole spectrum, at $4.7 \sigma$ and $5.2 \sigma$ levels by the two groups, while the measurements using the hydrogen lines showed only significance levels below $3 \sigma$.

Since the BOB Collaboration decided to consider a magnetic field to be detected with FORS 2 in a single observation only above the $5 \sigma$ level, and excluding the two previously known magnetic stars HD 46328 and HD 125823, we have detected with FORS 2 magnetic fields in four stars in our sample of 69 OB stars; these objects are CPD $-57^{\circ} 3509$, HD 164492C, HD 54879, and CPD $-62^{\circ} 2124$. This leads to a magnetic field detection rate of $6 \pm 3 \%$, which is compatible with the detection rate of $7 \%$ reported by Wade et al. (2014). However, CPD $-57^{\circ} 3509$ and CPD $-62^{\circ} 2124$ are both He-strong stars and thus strongly magnetic (e.g., Smith 1996). Also the magnetic star in the system HD $164492 \mathrm{C}$ is very likely He strong (González et al. 2017). For the 20 objects preselected on the 
basis of their low $v \sin i$, we obtain a magnetic field detection rate of $5 \pm 5 \%$. An in-depth discussion on the incidence of magnetic fields in all stars observed in our sample with both FORS 2 and HARPSpol will be presented in a forthcoming paper.

We again compared the results of the independent reduction and analysis carried out by two teams using different and independent tools and pipelines and we were able to show that the results agree with expected statistical distributions. This gives us high confidence of the accuracy of our longitudinal magnetic field measurements.

The results presented in this article underline the central role of FORS 2 observations for stellar magnetism studies in stars of different spectral classification at almost all stages of stellar evolution, especially for fainter targets.

Acknowledgements. L.F. acknowledges financial support from the Alexande von Humboldt Foundation. A.K. acknowledges financial support from RFBR grant 16-02-00604A. T.M. acknowledges financial support from Belspo for contract PRODEX Gaia-DPAC. M.F.N. acknowledges support by the Austrian Science Fund (FWF) in the form of a Meitner Fellowship under project number N-1868-NBL. M.S. and S.H. thank Thomas Szeifert for providing the pipeline for the FORS spectra extraction. This research has made use of the SIMBAD and ViZieR databases.

\section{References}

Aerts, C., \& Rogers, T. M. 2015, ApJ, 806, L33

Appenzeller, I., Fricke, K., Fürtig, W., et al. 1998, The Messenger, 94, 1

Bagnulo, S., Szeifert, T., Wade, G. A., Landstreet, J. D., \& Mathys, G. 2002, A\&A, 389, 191

Bagnulo, S., Landolfi, M., Landstreet, J. D., et al. 2009, PASP, 121, 993

Bagnulo, S., Landstreet, J. D., Fossati, L., \& Kochukhov, O. 2012, A\&A, 538, A129

Bagnulo, S., Fossati, L., Landstreet, J. D., \& Izzo, C. 2015, A\&A, 583, A115

Beer, A. 1961, MNRAS, 123, 191

Blomme, R., Mahy, L., Catala, C., et al. 2011, A\&A, 533, A4

Bochkarev, N. G., \& Karitskaya, E. A. 2012, in From Interacting Binaries to Exoplanets: Essential Modeling Tools, eds. M. T. Richards, \& I. Hubeny, IAU Symp., 282, 75

Bouy, H., Huélamo, N., Martín, E. L., et al. 2009, A\&A, 493, 931

Briquet, M., Aerts, C., Baglin, A., et al. 2011, A\&A, 527, A112

Brott, I., de Mink, S. E., Cantiello, M., et al. 2011, A\&A, 530, A115

Buysschaert, B., Aerts, C., Bloemen, S., et al. 2015, MNRAS, 453, 89

Bychkov, V. D., Bychkova, L. V., \& Madej, J. 2009, MNRAS, 394, 1338

Carrier, F., North, P., Udry, S., \& Babel, J. 2002, A\&A, 394, 151

Castro, N., Fossati, L., Hubrig, S., et al. 2015, A\&A, 581, A81

Castro, N., Fossati, L., Hubrig, S., et al. 2017, A\&A, 597, L6

Dahm, S. E., Herbig, G. H., \& Bowler, B. P. 2012, AJ, 143, 3

Damiani, F., Flaccomio, E., Micela, G., et al. 2004, ApJ, 608, 781

De Becker, M., Rauw, G., Blomme, R., et al. 2005, A\&A, 437, 1029

Degroote, P., Briquet, M., Auvergne, M., et al. 2010, A\&A, 519, A38

Eikenberry, S. S., Chojnowski, S. D., Wisniewski, J., et al. 2014, ApJ, 784, L30

Ferrario, L., Pringle, J. E., Tout, C. A., \& Wickramasinghe, D. T. 2009, MNRAS, 400, L71

Fossati, L., Castro, N., Schöller M., et al. 2015, A\&A, 582, A45

Frémat, Y., Zorec, J., Hubert, A.-M., et al. 2002, A\&A, 385, 986

González, J. F., Hubrig, S., Przybilla, N., et al. 2017, MNRAS, 467, 437

Grunhut, J. H., \& Wade, G. A. 2013, in Setting a New Standard in the Analysis of Binary Stars, eds. K. Pavlovski, A. Tkachenko, \& G. Torres, EAS Pub. Ser., 64, 67

Heger, A., Woosley, S. E., \& Spruit, H. C. 2005, ApJ, 626, 350

Houk, N. 1994, in The MK Process at 50 Years: A Powerful Tool for Astrophysical Insight, eds. C. J. Corbally, R. O. Gray, \& R. F. Garrison, ASP Conf. Ser., 60, 285

Howarth, I. D., \& Reid, A. H. N. 1993, A\&A, 279, 148

Howarth, I. D., Siebert, K. W., Hussain, G. A. J., \& Prinja, R. K. 1997, MNRAS, 284,265
Hubrig, S., Kurtz, D. W., Bagnulo, S., et al. 2004a, A\&A, 415, 661 Hubrig, S., Szeifert, T., Schöller, M., Mathys, G., \& Kurtz, D. W. 2004b, A\&A, 415,685

Hubrig, S., Schöller, M., Savanov, I., et al. 2009, Astron. Nachr., 330, 708

Hubrig, S., Ilyin, I., Briquet, M., et al. 2011, A\&A, 531, L20

Hubrig, S., Fossati, L., Carroll, T. A., et al. 2014, A\&A, 564, L10

Hubrig, S., Schöller, M., Fossati, L., et al. 2015, A\&A, 578, L3

Hubrig, S., Scholz, K., Hamann, W.-R., et al. 2016, MNRAS, 458, 3381

Ignace, R., Oskinova, L. M., \& Massa, D. 2013, MNRAS, 429, 516

Karitskaya, E. A., Bochkarev, N. G., Hubrig, S., et al. 2010, IBVS, 5950, 1

Kharchenko, N. V., Piskunov, A. E., Röser, S., et al. 2009, A\&A, 504, 681

Koen, C., \& Eyer, L. 2002, MNRAS, 331, 45

Le Bouquin, J.-B., Berger, J.-P., Lazareff, B., et al. 2011, A\&A, 535, A67

Lenzen, R., Hartung, M., Brandner, W., et al. 2003, SPIE Conf. Ser., 4841, 944

Maeder, A., \& Meynet, G. 2005, A\&A, 440, 1041

Mandel, I., \& de Mink, S. E. 2016, MNRAS, 458, 2634

Marchant, P., Langer, N., Podsiadlowski, P., Tauris, T. M., \& Moriya, T. J. 2016, A\&A, 588, A50

Mason, B. D., Wycoff, G. L., Hartkopf, W. I., et al. 2001, AJ, 122, 3466

Mermilliod, J. C. 2006, VizieR Online Data Catalog: II/168

Meynet, G., Eggenberger, P., \& Maeder, A. 2011, A\&A, 525, L11

Morel, T., Hubrig, S., \& Briquet, M. 2008, A\&A, 481, 453

Morel, T., Castro, N., Fossati, L., et al. 2014, The Messenger, 157, 27

Morel, T., Castro, N., Fossati, L., et al. 2015, in New Windows on Massive Stars,

eds. G. Meynet, C. Georgy, J. Groh, \& P. Stee, IAU Symp., 307, 342

Morrison, N. D., \& Conti, P. S. 1978, ApJ, 224, 558

Motch, C., Lopes de Oliveira, R., \& Smith, M. A. 2015, ApJ, 806, 177

Nasseri, A., Chini, R., Harmanec, P., et al. 2014, A\&A, 568, A94

Nazé, Y., Broos, P. S., Oskinova, L., et al. 2011, ApJS, 194, 7

Nazé, Y., Rauw, G., Sana, H., \& Corcoran, M. F. 2013, A\&A, 555, A83

Nazé, Y., Petit, V., Rinbrand, M. A, et al. 2014, ApJS, 215, 10

Neiner, C., Landstreet, J. D., Alecian, E., et al. 2012, A\&A, 546, A44

Oskinova, L. M., Todt, H., Ignace, R., et al. 2011, MNRAS, 416, 1456

Oskinova, L. M., Nazé, Y., Todt, H., et al. 2014, Nature Comm., 5, 4024

Petit, V., Owocki, S. P., Wade, G. A., et al. 2013, MNRAS, 429, 398

Pigulski, A., \& Pojmański, G. 2008, A\&A, 477, 917

Pittard, J. M., \& Parkin, E. R. 2010, MNRAS, 403, 1657

Pourbaix, D., Tokovinin, A. A., Batten, A. H., et al. 2004, A\&A, 424, 727

Przybilla, N., Fossati, L., Hubrig, S., et al. 2016, A\&A, 587, A7

Rauw, G., Morel, T., \& Palate, M. 2012, A\&A, 546, A77

Rauw, G., Nazé, Y., Spano, M., et al. 2013, A\&A, 555, L9

Rogers, T. M., Lin, D. N. C., McElwaine, J. N., \& Lau, H. H. B. 2013, ApJ, 772, 21

Rousset, G., Lacombe, F., Puget, P., et al. 2003, SPIE Conf. Ser., 4839, 140

Saesen, S., Briquet, M., \& Aerts, C. 2006, Commum. Asteroseismol., 147, 109

Sana, H., Le Bouquin, J.-B., Lacour, S., et al. 2014, ApJS, 215, 15

Sanz-Forcada, J., Franciosini, E., \& Pallavicini, R. 2004, A\&A, 421, 715

Schnerr, R. S., Verdugo, E., Henrichs, H. F., \& Neiner, C. 2006, A\&A, 452, 969

Schöller, M. 2007, New Astron. Rev., 51, 628

Schöller, M., Correia, S., Hubrig, S., \& Kurtz, D. W. 2012, A\&A, 545, A38

Simón-Díaz, S., \& Herrero, A. 2014, A\&A, 562, A135

Smith, K. C. 1996, Ap\&SS, 237, 77

Smith, M. A., Lopes de Oliveira, R., \& Motch, C. 2016, Adv. Space Res., 58, 782

Snik, F., Jeffers, S., Keller, C., et al. 2008, SPIE Conf. Ser., 7014, 70140O

Sota, A., Maíz Apellániz, J., Morrell, N. I., et al. 2014, ApJS, 211, 10

Stelzer, B., Flaccomio, E., Montmerle, T., et al. 2005, ApJS, 160, 557

Stickland, D. J., Lloyd, C., \& Koch, R. H. 1997, The Observatory, 117, 295

Telting, J. H., Schrijvers, C., Ilyin, I. V., et al. 2006, A\&A, 452, 945

Torrejón, J. M., Schulz, N. S., \& Nowak, M. A. 2012, ApJ, 750, 75

Trepl, L., Hambaryan, V. V., Pribulla, T., et al. 2012, MNRAS, 427, 1014

Tuthill, P., Lacour, S., Amico, P., et al. 2010, SPIE Conf. Ser., 7735, 773510

ud-Doula, A., \& Naze, Y. 2016, Adv. Space Res., 58, 680

ud-Doula, A., Owocki, S. P., \& Townsend, R. H. D. 2008, MNRAS, 385, 97

Wade, G. A., Petit, V., Grunhut, J., \& Neiner, C. 2014, arXiv e-prints

[arXiv: 1411.6165]

Wade, G. A., Neiner, C., Alecian, E., et al. 2016, MNRAS, 456, 2

Wang, J., Townsley, L. K., Feigelson, E. D., et al. 2008, ApJ, 675, 464

Wisniewski, J. P., Chojnowski, S. D., Davenport, J. R. A., et al. 2015, ApJ, 811, L26

Yoon, S.-C., Langer, N., \& Norman, C. 2006, A\&A, 460, 199 